\section{HMDS and Specimen Drying for SEM:}

\author{
Philip Oshel
}

Hexamethyldisilizane (HMDS) is an excellent method of chemical drying of hydrated specimens. There are several variables involved in its use, the most easily controlled being the number of transitional steps from $100 \%$ ethanol (EtOH) to $100 \%$ HMDS and the drying temperature,

Fixation and dehydration are the same for both HMDS and CPD. Once the specimen is in the final $100 \%$ ethanol, it must then be transferred to $100 \%$ HMDS through a graded series of ethanol-HMDS mixtures. This can follow one of four basic paths:
Ratio absEtOH : HMDS starting from $100 \% \mathrm{EtOH}$ going to $100 \%$ HMDS
1) $100 \% E \Rightarrow 1: 1 \Rightarrow 100 \% \mathrm{H}$
2) $100 \% E \Rightarrow 2: 1 \Rightarrow 1: 2 \Rightarrow 100 \% H$
3) $100 \% E \Rightarrow 2: 1 \Rightarrow 1: 1 \Rightarrow 1: 2 \Rightarrow 100 \% H$
4) $100 \% E \Rightarrow 3: 1 \Rightarrow 1: 1 \Rightarrow 1: 3 \Rightarrow 100 \% H$
(Extra gradations may be added as needed, for instance between the
$3: 1-1: 1$ and $1: 1-1: 3$ steps)

After the final transitional step, make 3 changes in HMDS (the last two steps can sometimes be skipped). Dry from the last 100\% HMDS step, or exchange with new $100 \%$ HMDS one final time then dry. There should be just enough HMDS in the container to cover the specimen, any more just increases the drying time.

Time in these steps will usually be the same as that used in the final $100 \%$ EtOH steps. However, the time can apparently be extended will little ill effect on the sample. Incomplete transition from EtOH to HMDS is a worse source of problems than extended time in HMDS.

Choice of steps is basically determined by sample density, and the permeability (to HMDS and ethanol) of the least permeable structures in the sample. Microorganisms can usually be done with the first series, animal

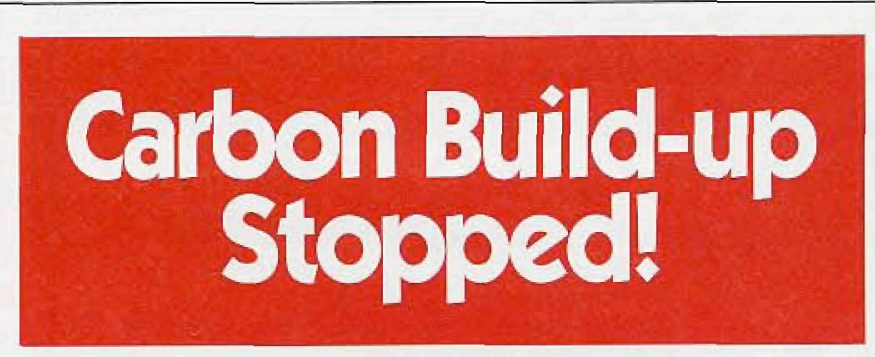

\section{SEM Laboratory Secret Revealed:}
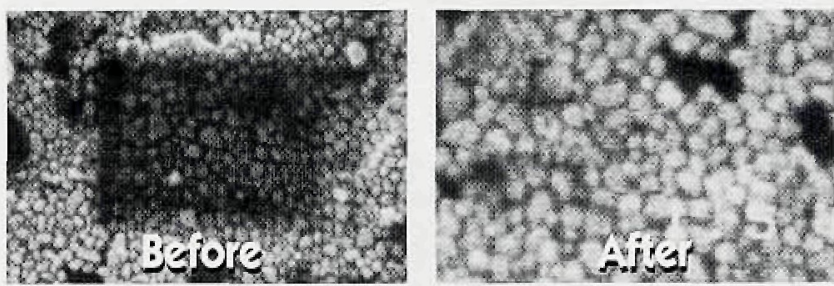

SEM manufacturers won't admit it, but most SEMs are subject to contamination build-up-even dry pumped systems. To stop hydrocarbon condensation, smart SEM users rely on the XEI Scientific SEM-CLEAN ${ }^{\top M}$ system

Result: Outstanding pictures at low $\mathrm{kV}$ and high resolution and no oil on EDS X-ray detector windows. The Nitrogen purge of the inexpensive SEM-CLEAN system cleans your electron microscope while you're away.

\section{SEM-CLEAN'" Stops the Oil XEI \\ S C I E N T F I C}

3124 Wessex Way, Redwood City, CA 94061

415-369-0133 - Fax 415-363-1659 tissues need the second or fourth series, and plants require the fourth series, or even more gradations because of their cell walls.

\section{Drying is done at either:}

$25^{\circ} \mathrm{C}=$ room temperature $8 \mathrm{hr} \Rightarrow$ overnight

$\left.37^{\circ} \mathrm{C}\right\}$

$\left.45^{\circ} \mathrm{C}\right\} 1=>4 \mathrm{hr}$

$\left.60^{\circ} \mathrm{C}\right\}$

(Drying time by both temperature and volume of fluid.)

The higher the temperature, the shorter the drying time, but the quality of results may also vary. Microscopic unicellular algae did best at $60^{\circ} \mathrm{C}$, fish skin at room temperature, bacieria equally well at room temperature and $60^{\circ} \mathrm{C}$.

HMDS may have a significant time advantage over CPD. If more specimens are being processed that can fit in the CPD chamber, then the times in the transitional fluids and for drying will be much less than the time necessary for $\mathrm{CPD}$. The greater the number of samples that can be batch processed, the greater the time advantage for HMDS.

HMDS has another advantage: if you can find containers that seal tightly enough (HMDS likes to evaporate given any chance at all), samples can be collected in the field, processed to $100 \%$ HMDS, then stored in vials filled with HMDS and transported long distances from remote sites - like from Antarctica to Chicago, Illinois. The samples are protected by the fluid, and at least for some specimens, so fewer artifacts than specimens stored in fixatives or alcohol. Dried specimens are of course fragile.

HMDS is not the cure-all for specimen drying. It can introduce it's own distorting artifacts and some samples, biological ones in particular, still shrink after drying as they do with CPD or freeze-drying. Some specimens do poorly when dried from HMDS. Plant tissues in particular may not do well, and may be better off dried by CPD. Also, if the specimen is going to be studied for elements that are labile, or in solution, standard fixation and dehydration methods won't work. Cryo techniques must be used, and if the specimen is to be examined in an unfrozen state, for example to look at structures and elements within cavities that would be obscured by ice if left hydrated, then the specimen must be dried by freeze-drying methods.

All of this information is empirical. Theoretical explanations and any modifications for particular samples are welcome!

A final note: HMDS must be used in a flume hood! A sniff of it will clear the sinuses back to the foramen magnum.

\section{MICIROSCOPIST}

The Conservation Analytical Laboratory (CAL) of the Smithsonian Institution, located in Suitland, MD, is recruiting for a Microscopist, GS-11 $(\$ 38,330$ $\$ 49,831)$ or GS-12 $(\$ 45,919-\$ 59,725)$. Duties include optical microscopy, chemical microscopy, freezingheating stage analysis, digital imaging and analysis, sample preparation, and microscope maintenance. The incumbent will be required to actively participate in the educational/training activities of CAL.

At least an undergraduate degree in physical/biological science or related field is required. The applicant must have knowledge of the principles and experience in the practice of optical microscopy, and skill in conducting training in a technical field to a professional audience.

For a copy of the vacancy announcement, call the Smithsonian 24-hour Automated Jobline, (202) 287 3102, press 9 and request Announcement \# 97PL3079. Applications must be postmarked by June 18, 1997

The Smithsonian Institution is an Equal Opportunity Employer. 